\title{
Metric Tensor and Christoffel Symbols based 3D Object Categorization
}

\author{
Syed Altaf Ganihar, Shreyas Joshi, Shankar Setty, Uma Mudenagudi \\ B. V. Bhoomaraddi College of Engineering and Technology
}

\begin{abstract}
In this paper we propose to address the problem of $3 \mathrm{D}$ object categorization. We model 3D object as a piecewise smooth Riemannian manifold and propose metric tensor and Christoffel symbols as a novel set of features. The proposed set of features captures the local and global geometry of $3 \mathrm{D}$ objects by exploiting the uniqueness and compatibility of the features. The metric tensor represents a geometrical signature of the $3 \mathrm{D}$ object in a Riemannian manifold. To capture global geometry we propose to use combination of metric tensor and Christoffel symbols, as Christoffel symbols measure the deviations in the metric tensor. The categorization of 3D objects is carried out using polynomial kernel SVM classifier. The effectiveness of the proposed framework is demonstrated on $3 \mathrm{D}$ objects obtained from different datasets and achieved comparable results.
\end{abstract}

\section{Introduction}

In this paper we propose three dimensional (3D) object categorization of a given 3D object using metric tensor and Christoffel symbols [1-4] with the help of kernel based support vector machine (SVM) classifier. With the availability of point cloud data of 3D objects, there is a surge of interest in novel methods for $3 \mathrm{D}$ object categorization. Categorization of $3 \mathrm{D}$ objects is a challenging problem. To address this issue we propose a set of features based on metric tensor and Christoffel symbols. Metric tensor together with Christoffel symbols captures the unique set of geometric features that are inherent to the $3 \mathrm{D}$ object shapes. The physical or intuitive parameter for an object is surface curvature. However they directly do not provide the inherent geometry of the 3D object [1-4]. One of the major challenges lies with the features to consider from the large datasets. Most of the 3D categorization methods use shapes, features and Bag-of-Words extracted from certain projections of the 3D objects. However, we propose to use features extracted from the geometry of 3D objects for categorization. The categorization of 3D objects finds its applications in the areas of content based retrieval, object detection, object recognition, and object tracking.

Humans usually are better in generic than in specific recognition, categorization is considered to be a much harder problem for computers. Since geometric features serves as a key for categorization, this influences the performance in terms of relevance and accuracy of results. Various sets of 3D features considered in literature for categorization and recognition are spin images [5], 3D shape 
context [6], global or local features [7-9], Point Feature Histogram (PFH) [10] and the Viewpoint Feature Histogram (VFH)model [11], Aspect Graph approach [12], spin images combined with other shape and contextual features [13]. In [14] the Global Structure Histogram (GSH) descriptor is presented to represent the point cloud information. The GSH represents objects such that it can generalize over different poses and views, and cope with incomplete data for correct categorization of objects. Use of viewpoints in all possible variations to build the training dataset and unsupervised approach for object categorization could be expensive during learning model building. In [15] authors propose a learning model by using a hypothetical 3D object category. Parts (collection of smaller image patches) of the objects are considered and correspondences between these parts are based on the appearance and geometric consistency. The final model is visualized as parts in a 3D graph based of the learned geometric relations. This approach classifies, localizes and does pose estimation of object in the image. In [16] authors present the work of multi-view part-based model of $[15,17]$, via minimal supervision and detection of objects under arbitrary or unseen poses. The proposed algorithm requires large number of views in training data in order to generalize which is an open issue.

In [18] 3D object categorization is introduced based on Bag-of-Words (BoW) paradigm. The visual vocabulary for bag of words is constructed in a multilevel way considering different seed regions. Hierarchical clustering is followed at each level for different region descriptors to obtain Bag-of-Words histograms for each mesh. Finally, the object categorization is done using one-against-all SVM classifier [19]. If the region descriptors are not properly clustered to obtain 3D visual words and if the vocabulary is so large that it could not distinguish between relevant and irrelevant variations may results into wrong categories. In [20] authors propose a discriminative approach to solve problem of 3D shape categorization. $3 \mathrm{D}$ local descriptors are extracted from 3D shapes, quantized using k-means to obtain 3D visual vocabulary and build a BoW representation. A general 3D Spatial Pyramid (3DSP) decomposition with multiple kernels is used to subdivide a cube impressed into surface of $3 \mathrm{D}$ shape repeatedly and compute weighted sum of histograms at increasingly fine sub-volumes. In [21] author expresses summary of categorization methods modeled from 2D images to 3D patch based model. In [22] author provides object detection from domestic scenes and based on shape model, categorizes the objects. The shape model is constructed by surface reconstruction method based on Growing Neural Gas (GNG) in combination with a shape distribution based descriptor. However, the shape ambiguity (eg. bowl and cup) between categories decreases the discrimination because of object similarities under certain perspectives. This requires large number of training data to be provided for improving the discriminative performance. In our approach we address the problem of 3D object categorization by proposing metric tensor and Christoffel symbols as geometric features of the 3D object using point cloud representation. Towards this we make the following contributions: 
1. We propose metric tensor and Christoffel symbols to represent basic geometry of 3D object which are intern used for 3D object categorization: we model $3 \mathrm{D}$ objects as a set of Riemannian manifolds and compute the features.

2. We propose framework for categorization of 3D objects using the proposed set of features, computed on local basis and captures the global geometry.

3. We demonstrate categorization of 3D objects using models obtained from state of art datasets like SHREC'12 using local patch based classification [23] and Princeton Shape benchmark dataset [24] using a BoW approach.

The rest of the paper is organized as follows. Section 2 describes the motivation and proposed approach. The geometric features are detailed in section 3 . We discuss proposed 3D object categorization in Section 4. We demonstrate the results on 3D object categorization in Section 5. Finally we conclude in Section 6.

\section{Motivation and Approach}

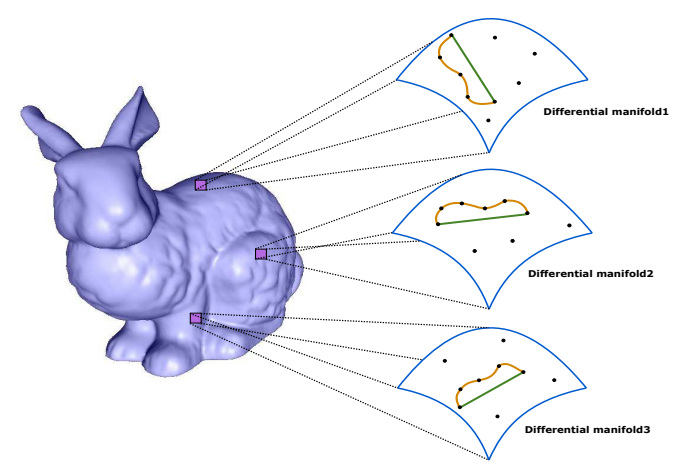

Fig. 1. The 3D object (bunny) exhibits non-uniformity in the distribution of geometrical properties and hence modeled as a set of Riemannian manifolds.

We model the given 3D object in a Euclidean space as a piecewise smooth Riemannian manifold. Let $V(x, y, z)$ be a $3 \mathrm{D}$ object in the Euclidean space and is modeled as,

$$
V(x, y, z) \mapsto \Psi(\mathcal{M}, g)
$$

where $\mathcal{M}, g$ represent the Riemannian manifold.

The piecewise smooth Riemannian manifold constitutes a continuous space and 3D point cloud represented in a Euclidean space is a sampled version of this continuous space. To capture the inherent geometry in Riemannian space, we need to model the $3 \mathrm{D}$ object as a piecewise smooth Riemannian manifold to account for the discontinuities in the geometry. A Riemannian manifold is a smooth construct which alone cannot represent the inherent geometry of the 3D 
object as the 3D object exhibits non-smooth behavior at certain positions due to non-uniform geometry. Consider the 3D model of a cube, the edge of the cube represents the transition in geometric properties and constitutes a non-smooth construct or is a geometric discontinuity. The derivatives for the Riemannian manifold are not defined at these points and contradicts the definition of a smooth Riemannian manifold. This necessitates the need of a piecewise smooth Riemannian manifold.

When we have a unique mapping of 3D Euclidean space to the Riemannian space, the claim is there exists a unique mapping in the discretized (sampled) version of the 3D Euclidean space to the Riemannian space. 3D point cloud in Euclidean space can be represented by a unique discretized piecewise smooth Riemannian manifold.

Riemannian manifold or Riemannian space $(\mathcal{M}, g)[2]$ is a real smooth differential manifold $\mathcal{M}$ equipped with an inner product $g_{p}$ on the tangent space at each point $\mathrm{p}$ and is given by,

$$
p \mapsto g_{p}(X(p), Y(p))
$$

where the mapping from $p \mapsto g_{p}$ is a smooth function with $X(p)$ and $Y(p)$ being vector fields in the tangent space of the $3 \mathrm{D}$ model at point $p$. The family $g_{p}$ of inner products is called Riemannian metric tensor.

The 3D object hence is a piecewise smooth differential manifold in which each tangent space is associated with an inner product which varies smoothly from point to point. The local geometrical properties of the 3D object can hence be inferred from the inner product computed on a local tangent plane for the object.

If $\mathcal{M}$ represents a differential manifold of dimensionality $n$ then a Riemannian metric [2] on the manifold $\mathcal{M}$ is a family of the inner products given by,

$$
g_{p}: T_{p} \mathcal{M} \times T_{p} \mathcal{M} \mapsto R \mid \forall p \in \mathcal{M}
$$

where $T_{p} \mathcal{M}$ represents the tangent space at each point $p$ on the manifold $\mathcal{M}$. The inner product $g_{p}$ on the manifold $\mathcal{M}$ defines a smooth function from $\mathcal{M} \mapsto R[1$, 2]. The definition of Riemannian metric tensor is dependent on the parametrization technique employed in the tangent plane of the differential manifold. Let $f$ denote the coordinate function defined on the tangent plane of the manifold which defines the map $f:(x, y, z) \mapsto(u, v)$, where $(u, v)$ defines the parameters on the tangent plane of the manifold. The parametrization of the manifold can be obtained by using the inverse map $f^{-1}$ to obtain the basis functions that can be used to compute the metric tensor on the manifold. The parametrization on the manifold is uniquely chosen for all the $3 \mathrm{D}$ objects so that the basis functions defined on the tangent plane are velocity vectors.

Given a Riemannian manifold $(\mathcal{M}, g)$ there exists a unique affine connection $\nabla$ on $\mathcal{M}$ that is symmetric and compatible with $g$ [2]. The uniqueness of the affine connection and the compatibility with the metric $g$ is described in Theorem 1. 
THEOREM 1 A Riemannian manifold $(\mathcal{M}, g)$ admits precisely one symmetric connection compatible with the metric. This particular connection is called the Riemannian connection or the LeviCivitta connection.

The affine connection $\nabla$ is called the LeviCivitta connection if

1. It preserves the metric i.e., $\nabla g=0$

2. It is torsion free. i.e., for any vector fields $X$ and $Y$ we have,

$$
\nabla_{X} Y-\nabla_{Y} X=[X, Y]
$$

$[X, Y]$ is the Lie Bracket [2] of the vector fields $X$ and $Y$.

The components of the LeviCivitta connection with respect to a system of local co-ordinates are called Christoffel symbols [2].

The Riemannian metric tensor along with Christoffel symbols for a defined parametrization of the tangent space constitute unique set of features for a given 3D object. But the definition of Riemannian metric tensor and Christoffel symbols depends on the choice of a co-ordinate system. To overcome this drawback we define a Cartesian world co-ordinate system in accordance with which we compute the components of metric tensor and Christoffel symbols. The given 3D objects under consideration are coarsely registered according to a predefined Cartesian world co-ordinate system using a ICP (iterative closest point) algorithm for 3D registration [25]. For every category of the 3D objects certain benchmark 3D objects are selected and the rest of the 3D objects are coarsely registered using the ICP algorithm.

In what follows we address the problem of 3D object categorization using a supervised learning approach. The input to the categorization framework is a 3D point cloud obtained either through set of images or from the modeling tools. The categorization is carried out using a supervised learning approach on a kernel based SVM framework. The geometric features for the input point cloud are computed and fed to the SVM. The geometric features used for the learning framework are metric tensor and Christoffel symbols. These set of features are used in a kernel based SVM framework for training and testing of the 3D objects. The testing data fed to the SVM which, after the learning process is able to classify the 3D object on a local patch basis.

\section{Features}

Riemannian metric tensor and Christoffel symbols effectively portray the inherent geometrical properties for a 3D object due to their compatibility and uniqueness and hence are best suited as features for categorization of $3 \mathrm{D}$ objects. The metric tensor represents the geometrical signature of the manifold in the local patch, however this alone may not capture the global geometrical properties since variations in the local patches are not inherently captured in the metric tensor. The Christoffel symbols give the numerical measure for the deviations in the geometrical properties of the manifold in the neighborhood of 
a patch. Hence variations in the modeled geometrical properties using metric tensor can be effectively captured by Christoffel symbols. The geometrical properties can quantitatively be represented using the combination of metric tensor and Christoffel symbols. The metric tensor and Christoffel symbols vary from point to point for a 3D object and hence can be represented as a mapping from the co-ordinates on the $3 \mathrm{D}$ object to the fields defined by metric tensor and Christoffel symbols and is given by,

$$
\Phi: V(x, y, z) \mapsto(g, \Gamma)
$$

where $\Psi(x, y, z)$ represents the given $3 \mathrm{D}$ object, $g$ and $\Gamma$ represent the metric tensor and Christoffel symbols. The 3D objects under consideration can be uniquely represented by the pair of fields $(g, \Gamma)$ defined on them.

\subsection{Metric Tensor}

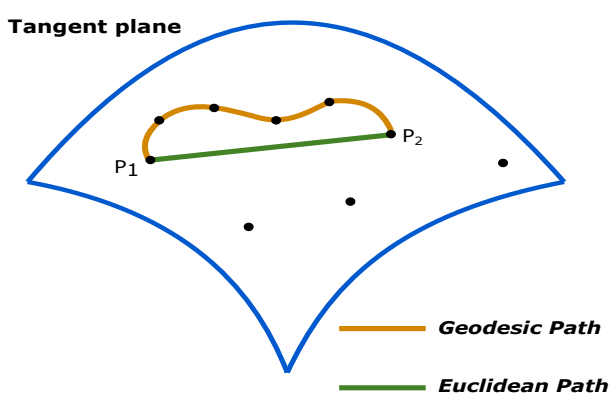

Fig. 2. The shortest distance between the points $P_{1}-P_{2}$ is computed as the geodesic distance (orange) on the manifold and is different from the Euclidean distance (green) between the points due to curvilinear properties of the surface.

Riemannian metric tensor is one of the features used for the classification of the models in the proposed method. The metric $g$ on a manifold is a second order covariant tensor field. The metric tensor $g_{\mu \nu}$ is a symmetric tensor and in 3 -dimensions comprises of 6 independent components. The metric tensor gives the quantitative measure for the deviation in the manifold from the Euclidean space. The inner product or the arclength of a curve in the manifold can be computed with the help of the metric tensor and is given by,

$$
d s^{2}=\sum_{\mu=1}^{3} \sum_{\nu=1}^{3} g_{\mu \nu} d x^{\mu} d x^{\nu}
$$

where $d s^{2}$ is the arclength of an infinitesimal curve on the manifold and $d x^{\mu}$, $d x^{\nu}$ are the contravariant tangent vectors in the tangent plane of the manifold. 
The deviation in the arclength $d s^{2}$ from the Euclidean distance function as shown in Fig. 2, gives a measure of the metric tensor $g$. To compute the metric tensor we calculate the arclength $d s^{2}$ as the geodesic distance between two neighboring points on a local patch as shown in Fig 3. The geodesic distance is computed on the $3 \mathrm{D}$ point cloud by using Algorithm 1 .

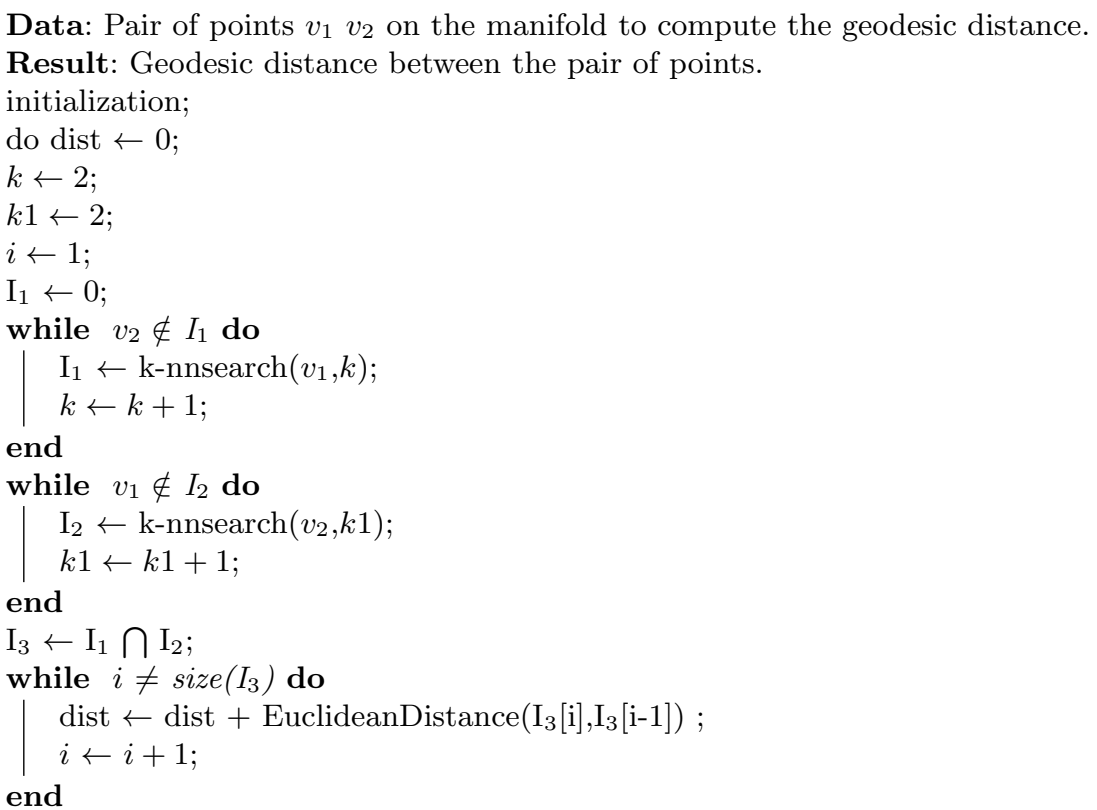

Algorithm 1: Geodesic distance computation on a pair of points on a point cloud.

In matrix notation the relation between the arclength $d s$ and components of the metric tensor $g$ is given by,

$$
d s^{2}=\left(\begin{array}{lll}
d x^{1} & d x^{2} & d x^{3}
\end{array}\right)\left(\begin{array}{lll}
g_{11} & g_{12} & g_{13} \\
g_{21} & g_{22} & g_{23} \\
g_{31} & g_{32} & g_{33}
\end{array}\right)\left(\begin{array}{l}
d x^{1} \\
d x^{2} \\
d x^{3}
\end{array}\right)
$$

The metric tensor consist of 6 independent components in 3-dimension. To compute the components of metric tensor a minimum of 6 pair of points are required for which the geodesic distances has to be computed. The geodesic distance is computed for 6 pair of points on the tangent plane of the manifold using Algorithm 1. Equation 7 is used to solve for the components of the metric tensor by using the 6 geodesic distances and the contravariant vectors $d x_{\mu}$ and $d x_{\nu}$ in the tangent plane. 


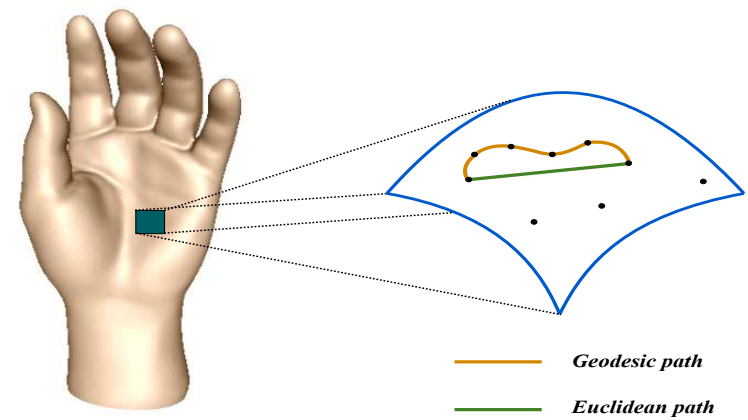

Fig. 3. The metric computation for a 3D object is carried out on a local tangent plane by computing the deviation of the geodesic distance from the Euclidean distance.

\subsection{Christoffel Symbols}

The Christoffel symbols give a measure of the deviation of the metric tensor as a function of position. The Christoffel symbols in 3-dimensions comprises of 18 independent components. The relation between metric tensor and Christoffel symbols is given by,

$$
\Gamma_{\mu \nu}^{\sigma}=\frac{1}{2} \sum_{\rho=1}^{3} g^{\sigma \rho}\left\{\frac{\partial g_{\rho \mu}}{\partial x^{\nu}}+\frac{\partial g_{\rho \nu}}{\partial x^{\mu}}-\frac{\partial g_{\mu \nu}}{\partial x^{\rho}}\right\}
$$

Equation 8 suggests that the computation of Christoffel symbols is dependent on the first derivative of metric tensor. The derivative operator in non-Euclidean space does not preserve the tensorial attributes of Christoffel symbols. They preserve the tensorial attributes under certain non-linear transformations. Equation 8 provides a pseudo-tensor which is utilized as one of the features in the proposed $3 \mathrm{D}$ object categorization.

The Christoffel symbols are computed from the metric tensor values for every 12 pair of points belonging to two neighboring tangent planes on the manifold. The computation of the Christoffel symbols for a pair of neighboring tangent planes is as shown in Fig 4. The Christoffel symbols represent the deviations in the metric tensor from one tangent plane to another due to the phenomenon of parallel transport on a curvilinear surface $[3,4]$.

The explicit geometry of the 3D object is represented by Riemannian curvature tensor which is captured by the combination of metric tensor and Christoffel symbols [2]. The uniqueness and compatibility of the metric tensor and Christoffel symbols as described in Theorem 1 enables us to model the given 3D object uniquely. Therefore metric tensor and Christoffel symbols can be used as geometrical features for the categorization of $3 \mathrm{D}$ objects. 


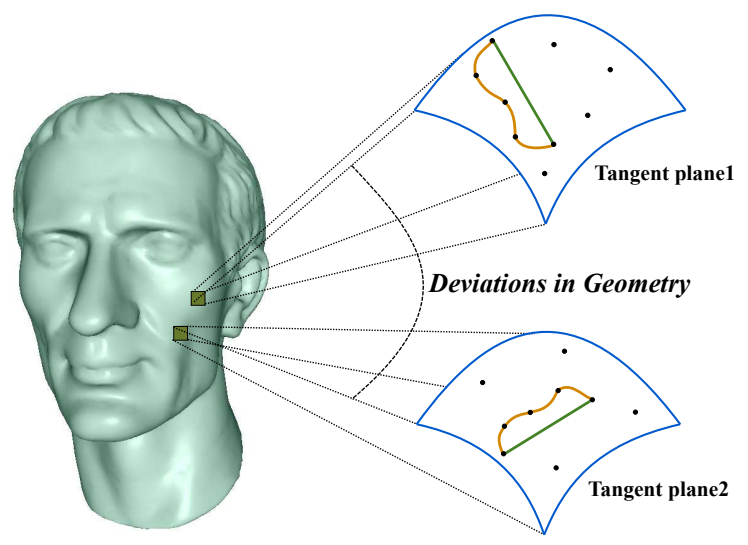

Fig. 4. The computation of Christoffel symbols for a 3D object is carried out on a pair of neighboring local tangent planes by computing the deviations in the metric tensor over the tangent planes.

\section{Categorization of $3 \mathrm{D}$ objects}

The categorization of the $3 \mathrm{D}$ objects into generic classes is carried out using a SVM framework with the proposed set of features and is shown in Fig 5. The proposed set of features are computed for a predefined set of models belonging to a particular class of objects and are fed to the SVM for learning.

\subsection{Learning Framework}

The categorization of 3D objects is carried out using a support vector machine framework [19]. The support vector machine is best suited for the categorization problem as it maps the features for classification into multidimensional vector space and supports non-linear kernels for classification. The features used for the categorization comprise of 24 independent components which are in turn dependent on the geometrical position of the point over which the features are computed. The normalization of the features is carried out by utilizing the positional dependence of the features for the 3D objects. The features for the training dataset are computed for unit scaled models to compensate for the scale dependence of the features. In our case we employ the polynomial kernel for the learning framework in support vector machine as the features, metric tensor and Christoffel symbols exhibit positional dependence.

The features, metric tensor and Christoffel symbols for the input 3D model are computed using the proposed method. The features computed are fed to the SVM framework for categorization of the 3D object using local patch based and 


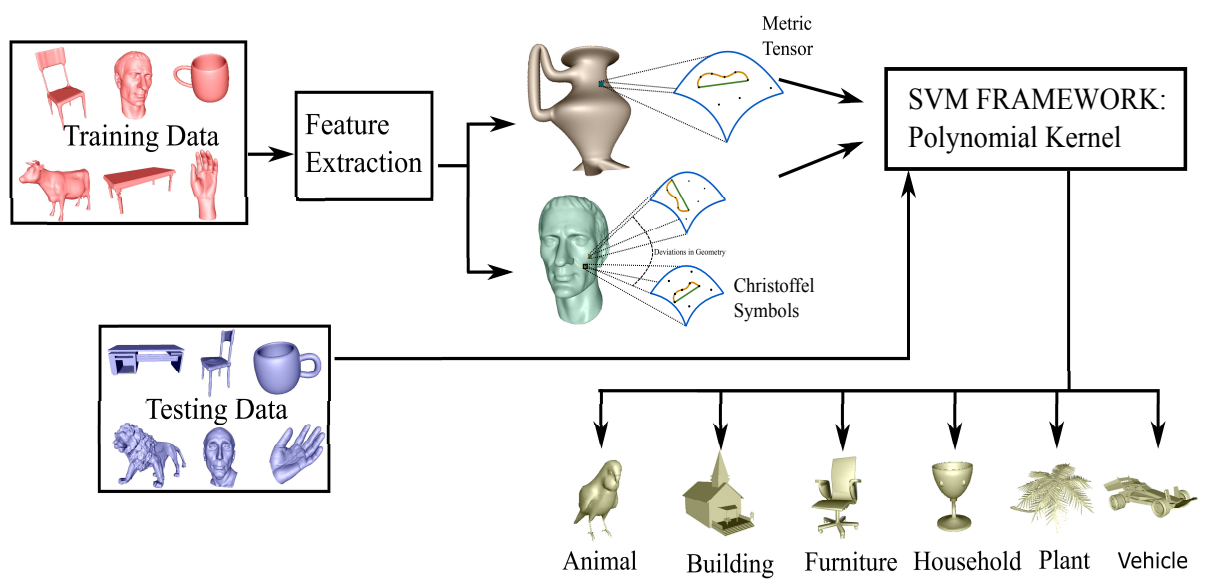

Fig. 5. Overview of the 3D object categorization.

BoW approach. In local patch based approach, the categorization of 3D objects is carried out into the predefined set of categories on a local patch basis with each patch comprising of 12 points. In BoW approach, k-means clustering is used for the proposed set of features on the training dataset to build the vocabulary. The vocabulary is used to compute the histogram on the proposed set of features. Based on the local patch or BoW approach SVM classifies the 3D objects into predefined set of categories.

\section{$5 \quad$ Results and discussions}

The effectiveness of the proposed categorization framework is demonstrated on models obtained from SHREC12 [23] and Princeton Shape Benchmark database [24] as shown in Fig 6. The algorithm is implemented on Intel(R) Core(TM) i74700MQ processor @ 2.40GHz and 8GB RAM with NVIDIA GeForce GT 755M graphics. The code is written in Matlab and $\mathrm{C}$ using point cloud library (PCL). We demonstrate the results for categorization of basic 3D objects in Section 5.1 and categorization of $3 \mathrm{D}$ objects in Section 5.2.

\subsection{Categorization of basic shapes}

The categorization framework is initially demonstrated on basic geometrical models like sphere, cone, cylinder and torus of varied scales. The global geometrical properties for basic shapes like sphere, cone and cylinder can be influenced from the local geometrical properties. The classification of the models is initially carried out on a local patch basis. The decision for the global classification of the model is derived from the results of the classification on the local basis. Table 1 shows the percentage content of each basic geometrical model in the testing 


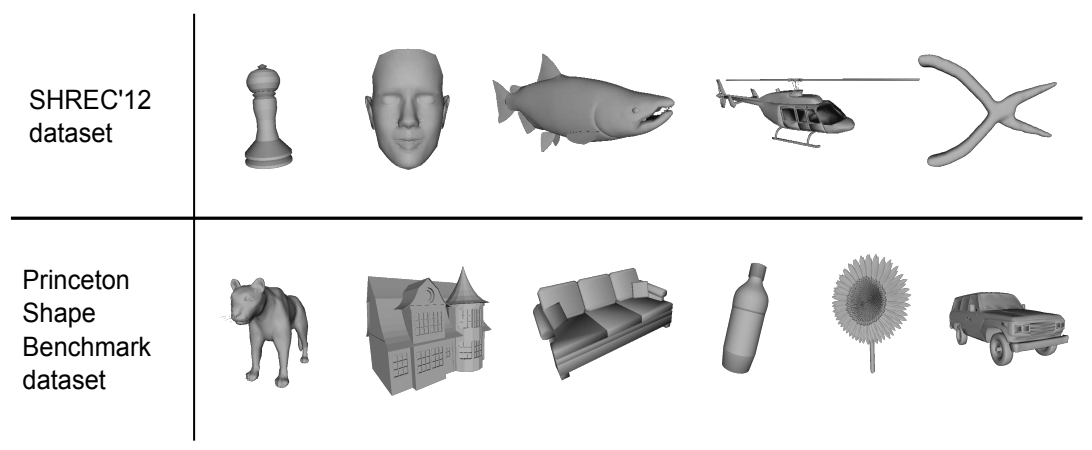

Fig. 6. Samples from the SHREC'12 and Princeton Shape Benchmark dataset

models for sphere, cone, cylinder and torus. We obtain an overall classification success rate of $100 \%$ for different scale models for each basic model. We also obtain an average accuracy of $83.60 \%$ for correct content categorization. The results demonstrates that the local patch exhibits similarity to the global structure for basic shapes and we make the following observations,

1. From the basic geometry it is clear that sphere exhibits more similarity to cone than cylinder and torus and is reinforced in the results.

2. Cone exhibits more similarity to cylinder and sphere than torus as cone is geometrically similar to both sphere and cylinder.

3. Cylinder exhibits more similarity to torus than sphere and cone due to the positive and null curvature regions in cylinder and torus.

4. Torus model is geometrically very similar to a sphere as it comprises of positive Gaussian curvature region in the exterior parts.

Table 1. Result for the categorization of basic models with percentage content.

\begin{tabular}{|c|c|c|c|c|}
\hline $\begin{array}{c}\text { Testing } \\
\text { data set }\end{array}$ & $\begin{array}{c}\text { Sphere } \\
\text { content }\end{array}$ & $\begin{array}{c}\text { Cone } \\
\text { content }\end{array}$ & $\begin{array}{c}\text { Cylinder } \\
\text { content }\end{array}$ & $\begin{array}{c}\text { Torus } \\
\text { content }\end{array}$ \\
\hline Sphere & $89.01 \%$ & $8.73 \%$ & $0.90 \%$ & $1.36 \%$ \\
\hline Cone & $7.31 \%$ & $85.19 \%$ & $6.50 \%$ & $0.99968 \%$ \\
\hline Cylinder & $0.367 \%$ & $0.027 \%$ & $91.79 \%$ & $7.82 \%$ \\
\hline Torus & $22.28 \%$ & $7.04 \%$ & $2.27 \%$ & $68.41 \%$ \\
\hline
\end{tabular}

\subsection{D Object Categorization}

We demonstrate the results for the categorization of the 3D objects using oneagainst-all testing strategy in SVM learning framework. We have used libSVM 
[26] for the training and testing of the SVM framework. The training and testing for the SHREC'12 dataset is carried out using local patch based approach with $3^{\text {rd }}$ order polynomial kernel based SVM framework. The SHREC'12 dataset consists of 20 models in each category. We have used 10 randomly selected models for training and the rest 10 models for testing. The overall accuracy for the SHREC'12 dataset is $66.42 \%$ measured as MCC (Mean Correct Classification) [20] for the 5 categories.

The Princeton shape benchmark dataset comprises of 1594 shapes for 6 classes in coarse level two. We have used coarse level two for the evaluation of the proposed framework for categorization. The subsets for training and testing proposed in [27] is used for the experimentation of the proposed categorization framework. The training and testing for the Princeton shape benchmark dataset is carried out using the BoW approach with $K=200$ for the proposed set of features. The training dataset used for the learning comprises of 807 models and the rest 787 models are used for testing using $15^{t h}$ order polynomial kernel based SVM classifier. The overall accuracy for the Princeton shape benchmark dataset is $67.90 \%$ for 6 categories. The confusion matrix for the SHREC'12 dataset and Princeton shape benchmark dataset is shown in Fig 7.

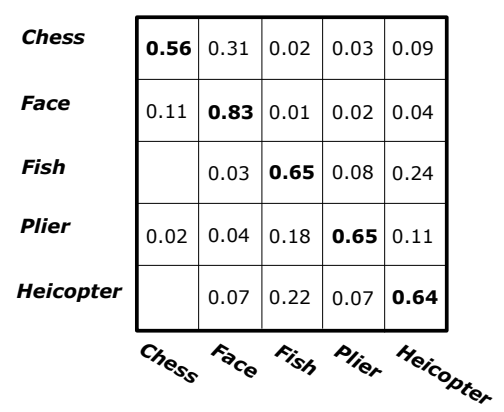

(a) SHREC'12 Dataset

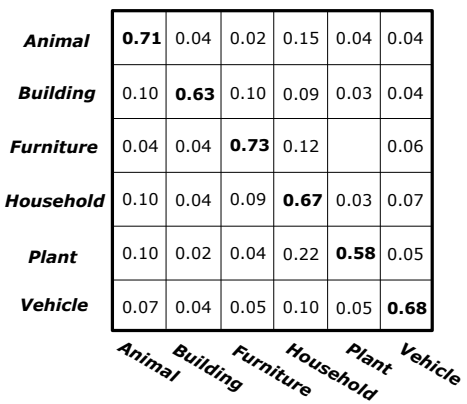

(b) Princeton Shape Benchmark Dataset

Fig. 7. Confusion Matrix for SHREC'12 and Princeton shape benchmark dataset

\subsection{Results Comparison}

The results for the 3D object categorization framework are carried out using the state of art datasets like SHREC'12 and Princeton shape benchmark dataset used by [20]. We compare our results with the results presented by [20] which uses a similar data set. For BoW approach with $\mathrm{K}=200$ and $\mathrm{L}=0$ the authors in [20] have achieved $61.43 \%$ measured as MCC with $3 \mathrm{DSP}-\chi^{2}$ kernel and for $\mathrm{K}=1000$ and $\mathrm{L}=1$ have achieved an accuracy of $66.31 \% \mathrm{MCC}$. With $\mathrm{K}=200$ for the BoW approach we have achieved an accuracy of $67.90 \%$ MCC. 


\section{Conclusion}

In this paper we have addressed the problem of 3D object categorization. We have modeled 3D object as a piecewise smooth Riemannian manifold and propose metric tensor and Christoffel symbols as a novel set of features. The metric tensor along with Christoffel symbols represents the inherent geometry of the $3 \mathrm{D}$ object uniquely in a Riemannian manifold and hence used to represent the global geometry for the $3 \mathrm{D}$ object. The categorization of $3 \mathrm{D}$ objects is carried out using polynomial kernel SVM classifier using proposed set of features with local patch based approach and BoW approach. We have demonstrated 3D objects categorization using SHREC'12 dataset and Princeton shape benchmark dataset.

\section{References}

1. Jost, J.: Riemannian Geometry and Geometric Analysis. Springer Universitat texts. Springer (2005)

2. Kumaresan, S.: A course in differential geometry and Lie groups. Texts and readings in mathematics. Hindustan Book Agency (2002)

3. Weinberg, S.: Gravitation and Cosmology: Principles and Applications of the General Theory of Relativity. Wiley, New York, NY (1972)

4. Misner, C., Thorne, K., Wheeler, J.: Gravitation. W.H. Freeman and Company (1973)

5. Johnson, A., Hebert, M.: Using spin images for efficient object recognition in cluttered 3d scenes. IEEE Transactions on Pattern Analysis and Machine Intelligence 21 (1999) 433 - 449

6. Frome, A., Huber, D., Kolluri, R., Bulow, T., Malik, J.: Recognizing objects in range data using regional point descriptors. In: Proceedings of the European Conference on Computer Vision (ECCV). (2004)

7. Papageorgiou, C., Poggio, T.: A trainable system for object detection. Int. J. Comput. Vision 38 (2000) 15-33

8. Schneiderman, H.: A Statistical Approach to 3D Object Detection Applied to Faces and Cars. PhD thesis, Robotics Institute, Carnegie Mellon University, Pittsburgh, PA (2000)

9. Viola, P.A., Jones, M.J.: Rapid object detection using a boosted cascade of simple features. In: CVPR (1). (2001) 511-518

10. Rusu, R.B., Blodow, N., Beetz, M.: Fast point feature histograms (fpfh) for 3d registration. In: Proceedings of the 2009 IEEE International Conference on Robotics and Automation. ICRA'09, Piscataway, NJ, USA, IEEE Press (2009) 1848-1853

11. Rusu, R.B., Bradski, G.R., Thibaux, R., Hsu, J.: Fast 3d recognition and pose using the viewpoint feature histogram. In: IROS, IEEE (2010) 2155-2162

12. Cyr, C.M., Kimia, B.B.: 3d object recognition using shape similarity-based aspect graph. In: ICCV. (2001) 254-261

13. Golovinskiy, A., Kim, V.G., Funkhouser, T.A.: Shape-based recognition of 3d point clouds in urban environments. In: ICCV, IEEE (2009) 2154-2161

14. Madry, M., Ek, C.H., Detry, R., Hang, K., Kragic, D.: Improving generalization for $3 \mathrm{~d}$ object categorization with global structure histograms. In: IROS, IEEE (2012) 1379-1386

15. Savarese, S., Li, F.F.: 3d generic object categorization, localization and pose estimation. In: ICCV, IEEE (2007) 1-8 
16. Savarese, S., Li, F.F.: Multi-view object categorization and pose estimation. In Cipolla, R., Battiato, S., Farinella, G.M., eds.: Computer Vision: Detection, Recognition and Reconstruction. Volume 285 of Studies in Computational Intelligence. Springer (2010) 205-231

17. Savarese, S., Li, F.F.: View synthesis for recognizing unseen poses of object classes. In Forsyth, D.A., Torr, P.H.S., Zisserman, A., eds.: ECCV (3). Volume 5304 of Lecture Notes in Computer Science., Springer (2008) 602-615

18. Toldo, R., Castellani, U., Fusiello, A.: A bag of words approach for 3d object categorization. In: Proceedings of the 4th International Conference on Computer Vision/Computer Graphics CollaborationTechniques. MIRAGE '09, Berlin, Heidelberg, Springer-Verlag (2009) 116-127

19. Burges, C.J.: A tutorial on support vector machines for pattern recognition. Data Mining and Knowledge Discovery 2 (1998) 121-167

20. Lpez-Sastre, R.J., Garca-Fuertes, A., Redondo-Cabrera, C., Acevedo-Rodrguez, F.J., Maldonado-Bascn, S.: Evaluating 3d spatial pyramids for classifying 3d shapes. Computers Graphics 37 (2013) 473-483

21. Pinz, A.: Object categorization. Found. Trends. Comput. Graph. Vis. 1 (2005) 255-353

22. Mueller, C.A.: 3D Object Shape Categorization in Domestic Environments. Technical Report February, Bonn-Rhein-Sieg University, Sankt Augustin, Germany (2012)

23. Biasotti, S., Bai, X., Bustos, B., Cerri, A., Giorgi, D., Li, L., Mortara, M., Sipiran, I., Zhang, S., Spagnuolo, M. In Spagnuolo, M., Bronstein, M.M., Bronstein, A.M., Ferreira, A., eds.: 3DOR, (Eurographics Association) 101-107

24. : The princeton shape benchmark. In: Proceedings of the Shape Modeling International 2004. SMI '04, Washington, DC, USA, IEEE Computer Society (2004) $167-178$

25. Rusinkiewicz, S., Levoy, M.: Efficient variants of the ICP algorithm. In: Third International Conference on 3D Digital Imaging and Modeling (3DIM). (2001)

26. Chang, C.C., Lin, C.J.: Libsvm: A library for support vector machines. ACM Trans. Intell. Syst. Technol. 2 (2011) 27:1-27:27

27. Knopp, J., Prasad, M., Willems, G., Timofte, R., Van Gool, L.: Hough transform and 3d surf for robust three dimensional classification. In Daniilidis, K., Maragos, P., Paragios, N., eds.: Computer Vision ECCV 2010. Volume 6316 of Lecture Notes in Computer Science. Springer Berlin Heidelberg (2010) 589-602 